\title{
OP-0092 - Clinical proven Standardised and synergistic formula for newly diagnosed type II diabetics
}

Komal S. Patel ${ }^{(1)}$, Dr. Lal Hingorani( ${ }^{(2)}$, Dr. Vineet C. Jain ${ }^{(3)}$

(1) Parul Institute of Pharmacy, Parul University, P.O Limda, Waghodia, Vadodara-391760.

(2) Managing Director, Pharmanza herbal Pvt. Ltd., Dharmaj, Anand- 388430.

Background:

(3) Professor, Bhagvan Mahavir College of Pharmacy, Surat, Gujarat.

- Diabetes mellitus is an endocrinological metabolic disorder characterized by elevated blood glucose level due to impaired insulin secretion or cellular insensitivity.

- The most disturbing trend of this disease is that the age of onset has preceded by 10 years.

- Long term uncontrolled hyperglycemia serves as a bridge between diabetes and secondary complications, and so patients diagnosed at younger age are at greater risk of diabetic complications.

- Diabetes related expenditure is almost Rs. 4,500/- (US \$ 120) per person and therefore estimated annual cost of Rs. 180,000 million is a huge economic burden for developing country like India.

- Ayurvedic remedies for diabetes are usually a combination of multiple herbs with numbers of phytoconstituents with different potential action, which exerts it's effect by synergistic, potentiative and agonostic action.

- The formulation was prepared by Combining Momordica charantia, Gymnema sylvestre, Tinospora cordifolia, Withania somnifera, Cinnamomum zeylanicum and Curcuma longa.

- A developed formula contain various herbs which lowers blood sugar level by different mechanisms which coupled with immunomodulatory, detoxicant, antioxidant herbs.

\section{Aim:}

The primary objective of our study to evaluate effectiveness of standardise formulation for the initial treatment of diabetes to that of Metformin and assess change in lipid profile.

Method:

Study Design: Non-randomized, open labelled, parallel study

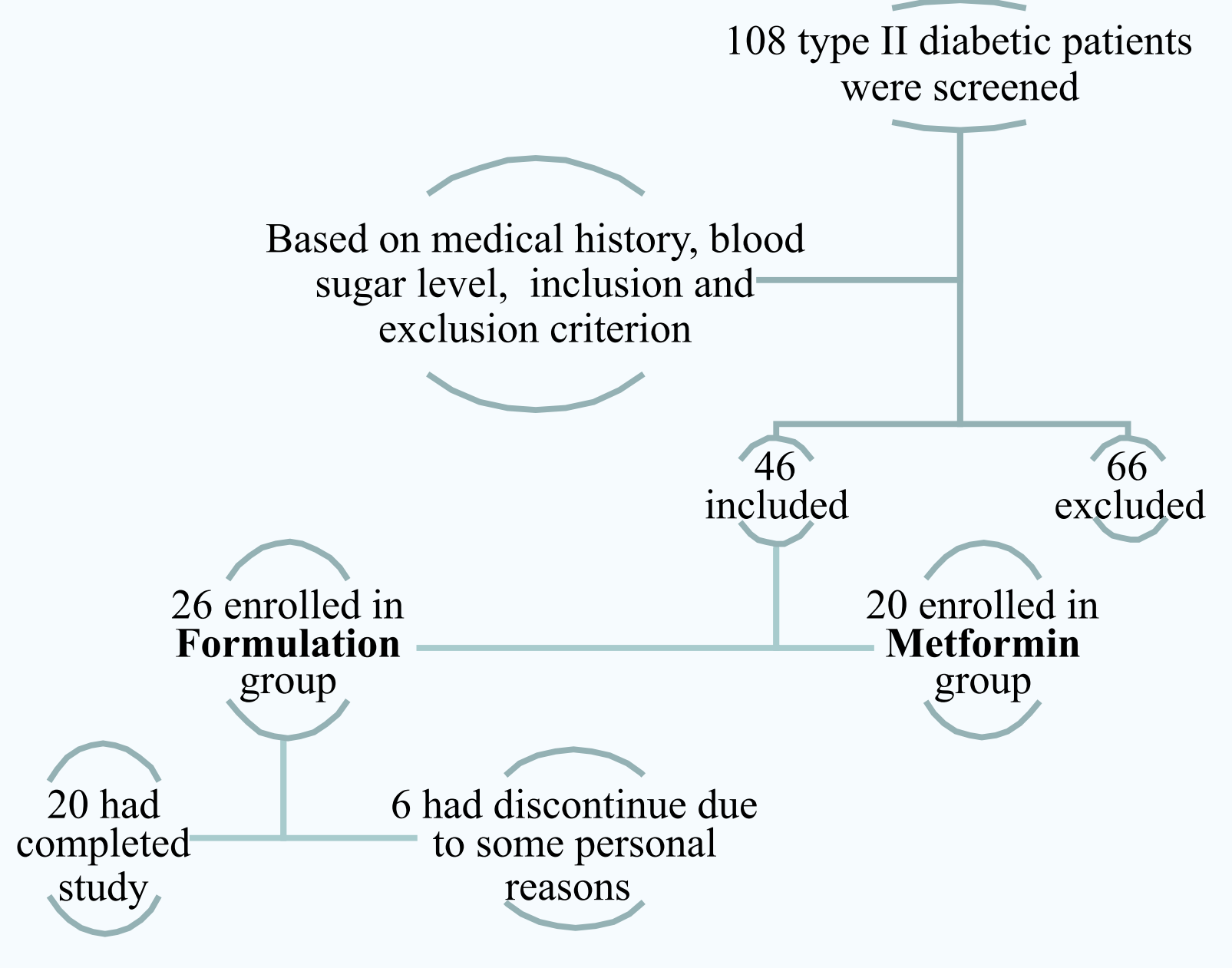

- All the investigations and laboratory parameters; CBC, FBS, PP2BS, HbA1C, LP, LFT, KFT were estimated before commencement of study as baseline and at the end of each month up to 3 months.

- Follow up visits at interval of $30 \pm 3$ days.

- Formulation group: 2 tablets of $750 \mathrm{mg}$ thrice a day Metformin group: 1 tablet of $1000 \mathrm{mg}$ once a day

\section{Result}

Mean age
Male : $48.6 \pm 11.2$

Female : $46.9 \pm 10.1$

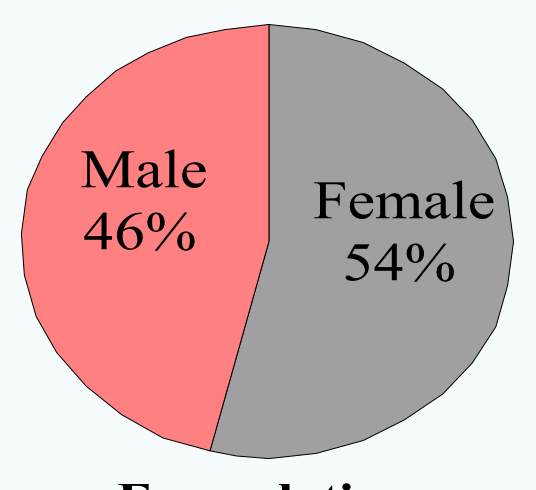

Formulation

Effect of Formulation and Metformin on Blood Glucose Level
Male : $49 \pm 8.3$

Female : $49.9 \pm 6.1$

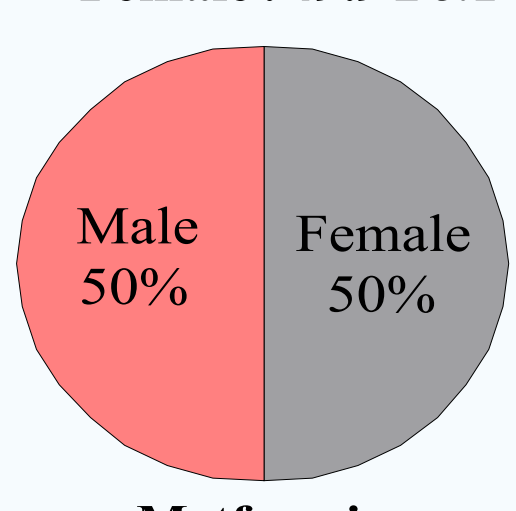

Metformin

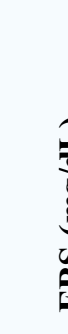

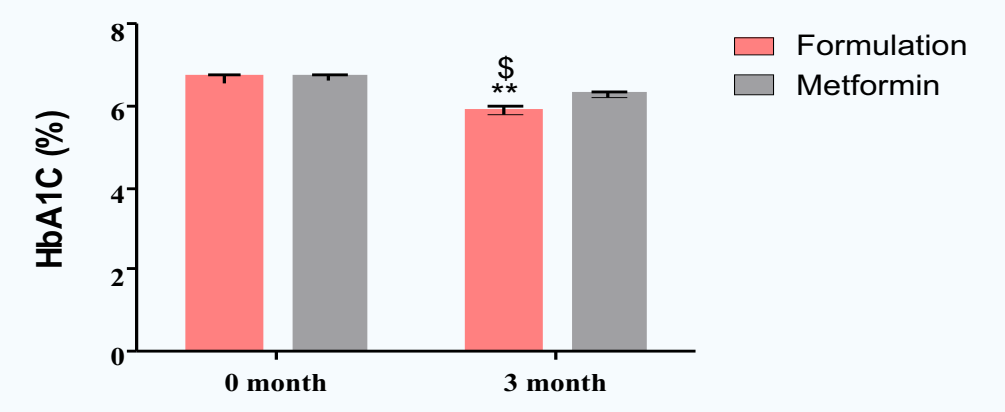

Effect of Formulation and Metformin on Lipid Profile


Each points shows mean \pm SEM, $n=20$. Data were analysed using two way ANOVA followed by Bonferroni post test, ${ }^{* *}$ shows significant difference from metformin group at the end of 3 rd month $(p<0.01)$. \$ shows significant difference at the end of month third compared to first month $(\mathrm{p}<0.001)$.

\begin{tabular}{|l|l|l|}
\hline \multirow{2}{*}{ Parameter } & \multicolumn{2}{c|}{ \% Reduction at the end of third month } \\
\cline { 2 - 3 } & \multicolumn{1}{|c|}{ Formulation Treated Group } & Metformin Treated Group \\
\hline FBS (mg/dL) & $32.61 \pm 6.30$ & $18.61 \pm 2.25$ \\
\hline PP2BS (mg/dL) & $40.48 \pm 15.30$ & $29.06 \pm 6.02$ \\
\hline HbA1C (\%) & 13.73 & 6.68 \\
\hline TC (mg/dL) & $42.72 \pm 17.80$ & $11.75 \pm 7.56$ \\
\hline TG (mg/dL) & $37.79 \pm 14.32$ & $16.70 \pm 5.79$ \\
\hline HDL $(\mathbf{m g} / \mathbf{d L})$ & $8.89 \pm 3.37$ & $1.95 \pm 1.2$ \\
\hline LDL (mg/dL) & $31.24 \pm 15.35$ & $16.19 \pm 7.32$ \\
\hline VLDL (mg/dL) & $50.21 \pm 25.23$ & $17.76 \pm 10.06$ \\
\hline
\end{tabular}

\section{Conclusion:}

- The blood sugar level was controlled within 4-8 weeks depending upon the initial blood sugar level.

- On the basis of the results, it was clearly understood that developed formulation may act through pancreatic and extra pancreatic mechanism(s) and significantly controls blood sugar in fasting and post prandial state, reduced the glycated haemoglobin level and modulated the lipid profile in newly diagnosed diabetic patients. 